\title{
Curvas de normalidad con Doppler continuo en arteria umbilical durante el embarazo
}

\author{
Manuel Oswaldo Gómez Wilches*
}

RESUMEN: Se ha hecho un estudio analítico, prospectivo y cruzado en 505 embarazadas con recién nacido normal, desde la semana 24 a la 42 del embarazo. El objetivo fue determinar en la población de Málaga - España, los valores de los índices de resistencia de doppler continuo en cordón umbilical, tanto en la población general como por sexo, con un aparato Vasoflo 3 y sonda de 4 MHz. Los índices de resistencia disminuyen a medida que progresa el embarazo normal, sin que en este hecho influya el sexo fetal.

PALABRAS CLAVES: Doppler continuo, arteria umbilical, embarazo normal, parámetros normales.

SUMMARY: A prospective analytic cross-sectional study has been carried with 505 normal pregnant women and normal newborn, from 24 to 42 weeks of the pregnancy. Our objective was to determine, in the Malaga - Spain population, the values of the resistance indexes with continuous doppler in umbilical cord, in general population and in male and female newborns. We used an apparatus Vasoflo 3 , with $4 \mathrm{MHz}$ probe. All the resistance index decreased through the normal pregnancy, without influence of fetal sex.

KEY WORDS: Continuous Doppler, umbilical artery, normal pregnancy, normal parameters.

\section{Introducción}

Durante las tres últimas décadas se han publicado diversas técnicas para el control biofísico fetal, y que utilizadas sistemáticamente en la asistencia obstétrica diaria (ecografía, ecocardiografía, monitorización tococardiográfica, etc.), significan un progreso fundamental en Medicina Perinatal (1). Sin embargo, no permiten la determinación de los flujos sanguíneos en las cavidades cardíacas y en los grandes vasos fetales. Entre éstos, los vasos del cordón umbilical, cuya fisiología es bien conocida (2) han sido razonablemente los vasos dianas en cualquier estudio novedoso en relación con el estado de bienestar fetal.

El estudio de los flujos sanguíneos en el árbol vascular fetal mediante el Efecto Doppler es, en la actualidad, el procedimiento más reciente en incorporarse al diagnóstico fisiopatológico respectivo, en forma prometedora e inocua (3-4). Con este fin se han seguido dos vías: la determinación de volúmenes de flujo sanguíneo absoluto y el análisis de las curvas de velocidad del flujo arterial.

El estudio de los volúmenes de flujo es complejo, ya que se ven influidos por varios factores y su tecnología es costosa. Sus resultados no justifican por el momento su costo (5). Las Curvas de Velocidad de Flujo son más accesibles, y las fuentes de error son más reducidas y el instrumental es menos caro (6).

Gosling y King (7) en 1975 introdujeron el doppler para el estudio del sistema vascular periférico, y comunicaron por primera vez la valoración del Indice de

* Médico Ginecólogo y Obstetra. Hospital Materno Infantil. Hospital Regional de Málaga-España. Servicio Andaluz de Salud.
Pulsatilidad con base en la información de la variación de FFT (Transformación Rápida de Fourier, que proporciona un análisis doppler = Análisis de Variación).

El empleo de la técnica doppler para estudios fetales fue comunicada por primera vez en 1977, cuando Fitzgerald y Drumm (8) demostraron las formas de onda de cambios de frecuencia del sistema doppler en la circulación arterial umbilical, y desde entonces se han acumulado muchos datos que indican que su uso es factible para el estudio de diversos componentes de la circulación fetal: aorta, corazón y cerebro.

En 1983, Campbell y col. (9) publicaron en forma más objetiva la morfología de onda velocimétrica en el sistema doppler en la circulación útero-placentaria (Arteria uterina, arteria arcuata y lecho placentario), y lo incluyen como nuevo método de gran ayuda para el diagnóstico de la condición fetal en la obstetricia moderna.

El empleo de la técnica Doppler en la obstetricia española se inició en 1986, con el grupo de Carrera y cols. $(1,10-13)$. Nuestro grupo de trabajo comenzó el estudio de la aplicación del Doppler dentro de la metodología diagnóstica materno-fetal en 1988, comenzando con el Doppler continuo en cordón umbilical (14-15).

\section{Objetivo}

El objetivo de este trabajo es establecer las curvas de normalidad de los diversos Indices de Resistencia de doppler continuo en arteria umbilical, durante el embarazo de curso normal, finalización a término y R.N. normal, en la población de Málaga, España. Los índices estudiados han sido: Stuart (S/D), Pourcelot (S-D/S), Pulsatilidad de Gosling (S-D/M) y el de conductancia de Milliez (D/S.100). 


\section{Material y métodos}

En la elaboración de este trabajo, he contado con una población de 1082 gestantes, de entre las atendidas en el Hospital Materno Infantil de Málaga, España durante los años de 1988 a 1991. En dichas gestantes se realizaron 2078 exploraciones de doppler continuo en cordón umbilical (Arteria umbilical), quedando finalmente para el estudio propuesto 505 pacientes, en las cuales se realizaron 970 exploraciones, y que cumplieron los siguientes requisitos, previamente establecidos:

a) Pacientes con embarazo de curso normal, sin patología que pudiese comprometer la unidad feto placentaria y alterar las medidas de Doppler Continuo en arteria umbilical.

b) Pacientes con parto a término, con terminación eutócica o tocúrgica por indicación obstétrica.

c) Pacientes con recién nacido normal, es decir, con índice de Apgar al quinto minuto normal, peso adecuado a la edad gestacional, y ausencia de malformaciones, así como de morbilidad o mortalidad perinatal.

La exploración vascular en la arteria umbilical, fue realizada con un aparato deDoppler continuo Bidireccional, modelo VASOFLO-3 SONICAID, con análisis espectral de Fourier, sonda de exploración de $4 \mathrm{MHz}$, sin filtros de frecuencias bajas y con monitor a color.

La paciente se colocaba en una posición semi-incorporada (semi-fowler), tanto en decúbito supino como en decúbito lateral, con la precaución de no tardar más de diez minutos en el procedimiento (para obviar el efecto hipotensor en el caso del decúbito supino), realizándose una exploración ecográfica previa sistemática rápida, que nos ayuda a la comprobación dática con la biometría fetal, a la localización placentaria y su homogeneidad, así como la inserción y morfología funicular, y la observación cualitativa de las cámaras de líquido amniótico, que fue efectuada con un aparato de ultrasonido de tiempo real, modelo SONOLAYER-L SAL-32-B de TOSHIBA, con sonda sectorial de $3.5 \mathrm{MHz}$. A continuación se aplicaba la sonda del doppler continuo de $4 \mathrm{MHz}$ sobre la zona abdominal de la gestante y correspondiente a un trayecto de cordón umbilical flotante en líquido amniótico, y preferentemente el próximo a su extremo placentario, que nos servía para la búsqueda de la señal doppler identificada por el sonido, la frecuencia del pulso, y la morfología de la onda de flujo de la Arteria Umbilical registrada en la pantalla del monitor a color.

Cuando la señal era estable, con la onda pulsátil de flujo arterial, y el trazado continuo de flujo venoso umbilicales, y sin variaciones propias de los movimientos respiratorios fetales, se congelaba la imagen del monitor y se calculaba automáticamente el promedio de los índices de resistencia y la FCF correspondiente a por lo menos cinco ondas de flujo, según la técnica descrita por Trudinger, y a continuación se imprimía dicho registro para anexarlo al caso de estudio.

Se han calculado los índices de resistencia siguientes: S/D, Pourcelot S-D/D, Pulsatilidad S-D/M y el de Conductancia (D/S) 100. Se han realizado las curvas de normalidad correspondientes a cada índice de resistencia de doppler continuo en arteria umbilical, con los siguientes valores percentiles 5, 10, 50, 90, 95, así como, $\mathbf{n}, \mathbf{X}$, DS, 2DS, ES, Mediana, Máximo, Mínimo y Rango, tanto para la población global, como para sexo fetal masculino y femenino.

Para la realización del presente trabajo, se ha diseñado una base de datos, en D-Base III Plus, para la incorporación de todos los datos en relación con el estudio, y se ha utilizado el paquete estadístico SPSS + para el procesamiento estadístico de los datos.

\section{Resultados}

Se exponen en las tablas I a VII y en las gráficas 1 a 4.

Tabla I

INDICE S/D EN EMBARAZADAS NORMALES (POBLACION GENERAL)

\begin{tabular}{|c|c|c|c|c|c|c|c|c|c|c|c|c|c|c|}
\hline & $\mathbf{n}$ & $\mathbf{X}$ & DS & 2DS & ES & M & P5 & P10 & P50 & P90 & P95 & MAX & MIN & RANGO \\
\hline Menor que 25 & 5 & 4.680 & 1.152 & 2.304 & 0.512 & 5.040 & - & - & 5.040 & - & - & 6.130 & 3.120 & 3.010 \\
\hline 26 & 3 & 4.240 & 2.067 & 4.134 & 1.194 & 3.210 & - & - & 3.210 & - & - & 6.620 & 2.890 & 3.730 \\
\hline 27 & 3 & 3.073 & 0.341 & 0.682 & 0.197 & 3.100 & - & - & 3.100 & - & - & 3.400 & 2.720 & 0.680 \\
\hline 29 & 8 & 3.215 & 0.546 & 1.092 & 0.193 & 3.425 & - & - & 3.425 & - & - & 3.930 & 2.470 & 1.460 \\
\hline 30 & 4 & 3.323 & 0.418 & 0.836 & 0.209 & 3.270 & - & - & 3.270 & - & - & 3.870 & 2.880 & 0.990 \\
\hline 31 & 4 & 3.210 & 0.497 & 0.994 & 0.248 & 3.125 & - & - & 3.135 & - & - & 3.840 & 2.730 & 1.110 \\
\hline 33 & 11 & 2.850 & 0.480 & 0.960 & 0.145 & 2.820 & - & 2.056 & 2.820 & 3.534 & - & 3.560 & 1.990 & 1.570 \\
\hline 34 & 20 & 2.450 & 0.559 & 1.118 & 0.125 & 2.305 & 1.873 & 1.924 & 2.305 & 3.472 & 3.937 & 3.960 & 1.870 & 2.090 \\
\hline 35 & 15 & 2.555 & 0.393 & 0.792 & 0.101 & 2.650 & - & 1.972 & 2.650 & 3.178 & - & 3.430 & 1.930 & 1.500 \\
\hline 36 & 24 & 2.587 & 0.674 & 1.348 & 0.138 & 2.325 & 1.885 & 1.915 & 2.325 & 3.855 & 4.110 & 4.180 & 1.880 & 2.300 \\
\hline 37 & 52 & 2.472 & 0.412 & 0.824 & 0.057 & 2.395 & 1.900 & 1.976 & 2.395 & 3.191 & 3.271 & 3.400 & 1.820 & 1.580 \\
\hline 38 & 59 & 2.504 & 0.468 & 0.936 & 0.610 & 2.460 & 1.850 & 1.990 & 2.460 & 3.120 & 3.330 & 4.600 & 1.750 & 2.850 \\
\hline 42 & 30 & 2.205 & 0.294 & 0.588 & 0.540 & 2.160 & 1.840 & 1.950 & 2.160 & 2.567 & 2.993 & 3.240 & 1.730 & 1.510 \\
\hline
\end{tabular}

Valores estadísticos del índice S/D en total de embarazadas normales desde la semana 24 a la 42. 


\section{Discusiones}

Los valores de los índices de resistencia con doppler continuo en arteria umbilical, en gestantes de curso normal obtenidos en el presente trabajo, no difieren con respecto a los publicados de otros estudios similares (1621) (ver tablas VI-VII), aunque sí en el mayor número de nuestra casuística, tras la cual comprobamos la presencia del descenso secuencial cronológico de los mismos, así como la ausencia de influencia sobre dichos índices por el sexo fetal.

Pienso que estos valores obtenidos con Doppler continuo son perfectamente válidos como curvas de normali- dad, incluso si utilizamos con más frecuencia el Doppler pulsado, como de hecho está sucediendo desde que tenemos a nuestro alcance más modernos y costosos aparatos de Doppler-Duplex, ya que los valores obtenidos con el Doppler continuo son equiparables a los obtenidos con el Doppler pulsado, según nuestra experiencia personal y la de otros autores (22-25). En relación con la posible influencia de la frecuencia cardíaca fetal sobre los valores de los índices de resistencia de Doppler, nuestra experiencia personal es negativa, así como la de otros grupos de trabajo (25-28).

Actualmente, y aprovechando el gran volumen asistencial de una Maternidad de 8000 partos/año como

Tabla II

INDICE R.I. EN EMBARAZADAS NORMALES (POBLACION GENERAL)

\begin{tabular}{|c|c|c|c|c|c|c|c|c|c|c|c|c|c|c|}
\hline & $n$ & $\mathbf{X}$ & DS & 2DS & ES & $\mathbf{M}$ & P5 & P10 & P50 & P90 & P95 & MAX & MIN & RANGO \\
\hline Menor que 25 & 5 & 2.028 & 2.814 & 5.628 & 1.258 & 0.790 & - & - & 0.790 & - & - & 7.060 & 0.670 & 6.390 \\
\hline 26 & 3 & 0.713 & 0.104 & 0.208 & 0.060 & 0.680 & - & - & 0.680 & - & - & 0.830 & 0.630 & 0.200 \\
\hline 27 & 3 & 0.653 & 0.029 & 0.058 & 0.017 & 0.670 & - & - & 0.670 & - & - & 0.670 & 0.620 & 0.050 \\
\hline 28 & 3 & 0.693 & 0.076 & 0.152 & 0.044 & 0.660 & - & - & 0.660 & - & - & 0.780 & 0.640 & 0.140 \\
\hline 29 & 8 & 0.669 & 0.060 & 0.120 & 0.021 & 0.695 & - & - & 0.695 & - & - & 0.740 & 0.580 & 0.160 \\
\hline 30 & 4 & 0.668 & 0.051 & 0.102 & 0.025 & 0.665 & - & - & 0.665 & - & - & 0.730 & 0.610 & 0.120 \\
\hline 31 & 4 & 0.660 & 0.039 & 0.078 & 0.020 & 0.655 & - & - & 0.655 & - & - & 0.710 & 0.620 & 0.090 \\
\hline 32 & 5 & 0.614 & 0.029 & 0.058 & 0.013 & 0.620 & - & - & 0.620 & - & - & 0.650 & 0.580 & 0.070 \\
\hline 33 & 11 & 0.617 & 0.075 & 0.150 & 0.023 & 0.630 & - & 0.472 & 0.630 & 0.700 & - & 0.700 & 0.450 & 0.250 \\
\hline 34 & 20 & 0.545 & 0.077 & 0.154 & 0.017 & 0.520 & 0.441 & 0.452 & 0.520 & 0.687 & 0.709 & 0.710 & 0.440 & 0.270 \\
\hline 35 & 15 & 0.577 & 0.050 & 0.100 & 0.013 & 0.580 & - & 0.486 & 0.580 & 0.644 & - & 0.650 & 0.480 & 0.170 \\
\hline 36 & 24 & 0.573 & 0.088 & 0.176 & 0.018 & 0.550 & 0.453 & 0.460 & 0.550 & 0.725 & 0.745 & 0.750 & 0.450 & 0.300 \\
\hline 37 & 52 & 0.563 & 0.064 & 0.128 & 0.009 & 0.560 & 0.460 & 0.473 & 0.560 & 0.657 & 0.680 & 0.690 & 0.430 & 0.260 \\
\hline 38 & 59 & 0.564 & 0.065 & 0.130 & 0.008 & 0.560 & 0.450 & 0.480 & 0.560 & 0.650 & 0.680 & 0.760 & 0.410 & 0.350 \\
\hline 39 & 102 & 0.546 & 0.060 & 0.120 & 0.006 & 0.550 & 0.452 & 0.470 & 0.550 & 0.620 & 0.640 & 0.680 & 0.380 & 0.300 \\
\hline 40 & 87 & 0.539 & 0.066 & 0.132 & 0.0070. & 300.434 & 0.468 & 0.530 & 0.632 & 0.652 & 0.710 & 0.340 & 0.370 & \\
\hline 41 & 70 & 0.530 & 0.055 & 0.110 & 0.007 & 0.530 & 0.446 & 0.460 & 0.530 & 0.609 & 0.624 & 0.640 & 0.410 & 0.230 \\
\hline 42 & 30 & 0.517 & 0.050 & 0.100 & 0.009 & 0.515 & 0.439 & 0.470 & 0.515 & 0.569 & 0.636 & 0.680 & 0.400 & 0.280 \\
\hline
\end{tabular}

Valores estadísticos del índice de Pourcelot en total de embarazadas normales desde la semana 24 a la 42.

Tabla III

INDICE P.I. EN EMBARAZADAS NORMALES (POBLACION GENERAL)

\begin{tabular}{|c|c|c|c|c|c|c|c|c|c|c|c|c|c|c|}
\hline & $n$ & $\mathbf{X}$ & DS & 2DS & ES & M & P5 & P10 & P50 & $\mathbf{P 9 0}$ & P95 & MAX & MIN & RANGO \\
\hline Menor que 25 & 5 & 1.690 & 0.553 & 1.106 & 0.247 & 1.700 & - & - & 1.700 & - & - & 2.500 & 1.080 & 1.420 \\
\hline 26 & 3 & 1.217 & 0.310 & 0.620 & 0.179 & 1.090 & - & - & 1.090 & - & - & 1.570 & 0.990 & 0.580 \\
\hline 27 & 3 & 1.060 & 0.040 & 0.080 & 0.023 & 1.060 & - & - & 1.060 & - & - & 1.100 & 1.020 & 0.080 \\
\hline 28 & 3 & 1.277 & 0.247 & 0.494 & 0.142 & 1.160 & - & - & 1.160 & - & - & 1.560 & 1.110 & 0.450 \\
\hline 29 & 8 & 1.070 & 0.174 & 0.348 & 0.061 & 1.100 & - & - & 1.100 & - & - & 1.320 & 0.800 & 0.520 \\
\hline 30 & 4 & 1.098 & 0.137 & 0.274 & 0.068 & 1.070 & - & - & 1.070 & - & - & 1.280 & 0.970 & 0.310 \\
\hline 31 & 4 & 1.070 & 0.118 & 0.236 & 0.059 & 1.060 & - & - & 1.060 & - & - & 1.200 & 0.960 & 0.240 \\
\hline 32 & 5 & 1.072 & 0.266 & 0.522 & 0.119 & 1.050 & - & - & 1.050 & - & - & 1.520 & 0.850 & 0.670 \\
\hline 33 & 11 & 0.997 & 0.165 & 0.330 & 0.050 & 1.010 & - & 0.698 & 1.010 & 1.244 & - & 1.260 & 0.670 & 0.590 \\
\hline 34 & 20 & 0.845 & 0.217 & 0.434 & 0.048 & 0.750 & 0.583 & 0.644 & 0.750 & 1.148 & 1.445 & 1.460 & 0.580 & 0.880 \\
\hline 35 & 15 & 0.929 & 0.175 & 0.350 & 0.045 & 0.890 & - & 0.708 & 0.890 & 1.218 & - & 1.380 & 0.660 & 0.720 \\
\hline 36 & 24 & 0.913 & 0.238 & 0.576 & 0.049 & 0.850 & 0.583 & 0.630 & 0.850 & 1.330 & 1.485 & 1.510 & 0.580 & 0.930 \\
\hline 37 & 52 & 0.877 & 0.160 & 0.320 & 0.022 & 0.855 & 0.632 & 0.680 & 0.855 & 1.121 & 1.151 & 1.340 & 0.590 & 0.750 \\
\hline 38 & 59 & 1.031 & 0.913 & 0.826 & 0.119 & 0.880 & 0.590 & 0.680 & 0.880 & 1.180 & 1.570 & 7.740 & 0.530 & 7.210 \\
\hline 39 & 102 & 0.855 & 0.148 & 0.296 & 0.015 & 0.860 & 0.622 & 0.673 & 0.860 & 1.040 & 1.119 & 1.240 & 0.520 & 0.720 \\
\hline 40 & 87 & 0.841 & 0.213 & 0.426 & 0.023 & 0.810 & 0.598 & 0.648 & 0.810 & 1.050 & 1.096 & 2.210 & 0.430 & 1.780 \\
\hline 41 & 70 & 0.798 & 0.127 & 0.254 & 0.015 & 0.800 & 0.591 & 0.641 & 0.800 & 0.986 & 1.005 & 1.080 & 0.520 & 0.560 \\
\hline 42 & 30 & 0.777 & 0.135 & 0.270 & 0.025 & 0.755 & 0.612 & 0.660 & 0.755 & 0.977 & 1.142 & 1.290 & 0.590 & 0.700 \\
\hline
\end{tabular}

Valores estadísticos del índice de Pulsatilidad en total de embarazadas normales desde la semana 24 a la 42. 
Tabla IV

INDICE C.I. EN EMBARAZADAS NORMALES (POBLACION GENERAL)

\begin{tabular}{|c|c|c|c|c|c|c|c|c|c|c|c|c|c|c|}
\hline & $\mathbf{n}$ & $\mathbf{X}$ & DS & 2DS & ES & $\mathbf{M}$ & P5 & P10 & P50 & $\mathbf{P 9 0}$ & P95 & MAX & MIN & RANGO \\
\hline Menor que 25 & 5 & 27.53 & 14.95 & 29.90 & 6.686 & 25.68 & - & - & 25.68 & - & - & 50.56 & 10.90 & 39.62 \\
\hline 26 & 3 & 26.47 & 10.96 & 21.92 & 6.329 & 30.52 & - & - & 30.52 & - & - & 34.83 & 14.06 & 20.77 \\
\hline 27 & 3 & 33.13 & 2.246 & 4.492 & 1.297 & 33.05 & - & - & 33.05 & - & - & 35.42 & 30.93 & 4.490 \\
\hline 28 & 3 & 28.93 & 8.728 & 17.45 & 5.039 & 32.01 & - & - & 32.01 & - & - & 35.70 & 19.08 & 16.62 \\
\hline 29 & 8 & 31.53 & 5.708 & 11.41 & 2.018 & 28.80 & - & - & 28.80 & - & - & 40.12 & 24.80 & 15.32 \\
\hline 30 & 4 & 30.68 & 4.767 & 9.534 & 2.381 & 32.10 & - & - & 32.10 & - & - & 34.34 & 24.18 & 10.16 \\
\hline 31 & 4 & 32.71 & 3.668 & 7.336 & 1.834 & 33.25 & - & - & 33.25 & - & - & 36.25 & 28.09 & 8.160 \\
\hline 32 & 5 & 36.23 & 3.333 & 6.666 & 1.490 & 35.58 & - & - & 35.58 & - & - & 40.80 & 32.17 & 8.630 \\
\hline 33 & 11 & 36.38 & 7.133 & 14.26 & 2.151 & 35.75 & - & 28.61 & 35.75 & 50.56 & - & 52.55 & 28.57 & 23.98 \\
\hline 34 & 20 & 43.76 & 7.859 & 15.71 & 1.757 & 46.41 & 28.17 & 29.60 & 46.41 & 53.52 & 54.60 & 54.65 & 28.10 & 26.55 \\
\hline 35 & 15 & 42.05 & 6.503 & 13.00 & 1.679 & 40.80 & - & 33.60 & 40.80 & 52.84 & - & 54.34 & 32.17 & 22.17 \\
\hline 36 & 24 & 41.03 & 8.658 & 17.31 & 1.767 & 43.43 & 24.24 & 26.07 & 43.43 & 52.34 & 53.09 & 53.34 & 23.71 & 29.63 \\
\hline 37 & 52 & 42.45 & 7.111 & 14.22 & 0.986 & 43.04 & 29.72 & 31.97 & 43.04 & 51.62 & 54.01 & 63.09 & 28.24 & 34.85 \\
\hline 38 & 59 & 41.50 & 6.626 & 13.25 & 0.863 & 40.76 & 30.23 & 32.72 & 40.76 & 49.38 & 53.57 & 58.12 & 21.73 & 36.39 \\
\hline 39 & 102 & 43.64 & 6.132 & 12.26 & 0.607 & 43.28 & 33.82 & 36.23 & 43.28 & 51.42 & 53.12 & 60.57 & 27.55 & 33.02 \\
\hline 40 & 87 & 44.39 & 6.821 & 13.64 & 0.73145 & 6132.18 & 35.57 & 45.61 & 51.99 & 54.91 & 64.78 & 27.55 & 37.23 & \\
\hline 41 & 70 & 45.51 & 5.996 & 11.99 & 0.717 & 45.94 & 36.72 & 37.94 & 45.94 & 53.06 & 55.50 & 60.10 & 33.69 & 26.41 \\
\hline 42 & 30 & 46.30 & 5.230 & 10.46 & 0.955 & 47.22 & 33.31 & 41.11 & 47.22 & 51.31 & 54.23 & 57.73 & 28.62 & 29.11 \\
\hline
\end{tabular}

Valores estadísticos del índice de Conductancia en total de embarazadas normales desde la semana 24 a la 42.

Tabla V

INDICE S/D DOPPLER CONTINUO EN ARTERIA UMBILICAL

\begin{tabular}{|c|c|c|c|c|c|c|}
\hline \multirow[b]{3}{*}{$\begin{array}{l}\text { Año } \\
1989\end{array}$} & \multicolumn{6}{|c|}{ AUTORES } \\
\hline & $\begin{array}{l}\text { Stuart } \\
\text { et al } \\
(18)\end{array}$ & $\begin{array}{c}\text { Schulman } \\
\text { et al } \\
\text { (21) }\end{array}$ & $\begin{array}{l}\text { Trudinger } \\
\text { et al } \\
\text { (17) }\end{array}$ & $\begin{array}{l}\text { Fogarty } \\
\text { et al } \\
\text { (19) }\end{array}$ & $\begin{array}{c}\text { Santonja, } \\
\text { Armero } \\
\text { (20) }\end{array}$ & $\begin{array}{c}\text { Gómez, } \\
\text { Gallo } \\
(29)\end{array}$ \\
\hline & 1980 & $\begin{array}{l}1984 \\
1991\end{array}$ & & 1985 & & 1989 \\
\hline $\begin{array}{l}\text { Casos } \\
129\end{array}$ & 18 & $\begin{array}{c}89 \\
505\end{array}$ & & 15 & & 85 \\
\hline Determin & 182 & 162 & 100 & 783 & 185 & 970 \\
\hline Estudio & $\mathrm{L}$ & C & $\mathrm{L}$ & $\mathrm{L}$ & C & C \\
\hline Semanas & $20-40$ & $25-41$ & $25-41$ & $16-42$ & $20-43$ & $24-42$ \\
\hline Sonda & $5 \mathrm{MHZ}$ & $4 \mathrm{MHZ}$ & $4 \mathrm{MHZ}$ & $?$ & $4 \mathrm{MHZ}$ & $4 \mathrm{MHZ}$ \\
\hline Filtro & $?$ & $?$ & $300 \mathrm{HZ}$ & $200 \mathrm{HZ}$ & $10 \mathrm{HZ}$ & $10 \mathrm{HZ}$ \\
\hline X 24 & - & - & - & 2.58 & 3.81 & 4.680 \\
\hline X 28 & 3.75 & 2.70 & 3.20 & 3.16 & 3.08 & 3.623 \\
\hline X 32 & 3.00 & 2.60 & 2.85 & 2.82 & 3.04 & 2.860 \\
\hline X 34 & - & - & - & 2.64 & 2.96 & 2.450 \\
\hline X 36 & - & - & - & 2.45 & 2.62 & 2.587 \\
\hline X 38 & - & - & - & 2.34 & 2.52 & 2.504 \\
\hline X 40 & 2.20 & 2.35 & 2.10 & 2.25 & 2.50 & 2.334 \\
\hline X 42 & - & - & - & 2.53 & 2.25 & 2.205 \\
\hline
\end{tabular}

Resultados comparativos de diversos autores con relación al índice S/D con doppler continuo en arteria umbilical. 
Tabla VI

INDICE DE POURCELOT DOPPLER CONTINUO EN A.U.

\begin{tabular}{|c|c|c|c|c|}
\hline & \multicolumn{4}{|c|}{ A UTORES } \\
\hline & $\begin{array}{c}\text { Thompson } \\
\text { et al } \\
(16)\end{array}$ & $\begin{array}{c}\text { Fogarty } \\
\text { et al } \\
(19)\end{array}$ & $\begin{array}{c}\text { Santonja-Lucas, } \\
\text { Armero } \\
(20) \\
\end{array}$ & $\begin{array}{c}\text { Gómez, } \\
\text { Gallo } \\
(29)\end{array}$ \\
\hline Año & 1988 & 1989 & 1989 & 1991 \\
\hline Casos & 35 & 85 & 129 & 505 \\
\hline Determin & 241 & 783 & 185 & 970 \\
\hline Estudio & $\mathrm{L}$ & $\mathrm{L}$ & $\mathrm{C}$ & $\mathrm{C}$ \\
\hline Semanas & $20-40$ & $16-42$ & $20-43$ & $24-42$ \\
\hline Sonda & $4 \mathrm{MHZ}$ & $?$ & $4 \mathrm{MHZ}$ & $4 \mathrm{MHZ}$ \\
\hline Filtro & $?$ & $200 \mathrm{HZ}$ & $10 \mathrm{HZ}$ & $10 \mathrm{HZ}$ \\
\hline X 24 & 0.711 & 0.70 & 0.70 & 2.028 \\
\hline X 28 & 0.683 & 0.67 & 0.65 & 0.693 \\
\hline X 32 & 0.630 & 0.63 & 0.65 & 0.614 \\
\hline X 34 & 0.611 & 0.60 & 0.64 & 0.545 \\
\hline X 36 & 0.556 & 0.57 & 0.59 & 0.573 \\
\hline X 38 & 0.537 & 0.55 & 0.58 & 0.564 \\
\hline X 40 & 0.518 & 0.55 & 0.53 & 0.539 \\
\hline X 42 & - & 0.59 & 0.53 & 0.517 \\
\hline
\end{tabular}

Resultados comparativos de diversos autores con relación al índice de Pourcelot con doppler continuo en arteria umbilical.

Tabla VII

INDICE DE PULSATILIDAD DOPPLER CONTINUO EN A.U.

\begin{tabular}{|lccc|}
\hline & & A U T O R E S & \\
\cline { 2 - 4 } & Fogarty et al & Santonja, Armero & Gómez, Gallo \\
& $(19)$ & $(20)$ & $(29)$ \\
\cline { 2 - 4 } Año & 1989 & 1989 & 1991 \\
Casos & 85 & 129 & 505 \\
Determin & 783 & 185 & 970 \\
Estudio & $\mathrm{L}$ & $\mathrm{C}$ & $\mathrm{C}$ \\
Semanas & $16-42$ & $20-43$ & $20-42$ \\
Sonda & $?$ & $4 \mathrm{MHZ}$ & $4 \mathrm{MHZ}$ \\
Filtro & $200 \mathrm{HZ}$ & $10 \mathrm{HZ}$ & $10 \mathrm{HZ}$ \\
X 24 & 1.34 & 1.18 & 1.690 \\
X 28 & 1.14 & 1.02 & 1.277 \\
X 32 & 1.06 & 1.02 & 1.072 \\
X 34 & 0.97 & 1.01 & 0.845 \\
X 36 & 0.93 & 0.89 & 0.913 \\
X 38 & 0.87 & 0.87 & 1.031 \\
X 40 & 0.87 & 0.87 & 0.841 \\
X 42 & 1.00 & 0.79 & 0.777 \\
L $=$ Longitud. C= Cruzado & & & \\
\hline
\end{tabular}

Resultados comparativos de diversos autores con relación al índice de Pulsatilidad con doppler continuo en arteria umbilical. 
DOPPLER CONTINUO EN A. UMBILICAL- INDICE S/D (GLOBAL)

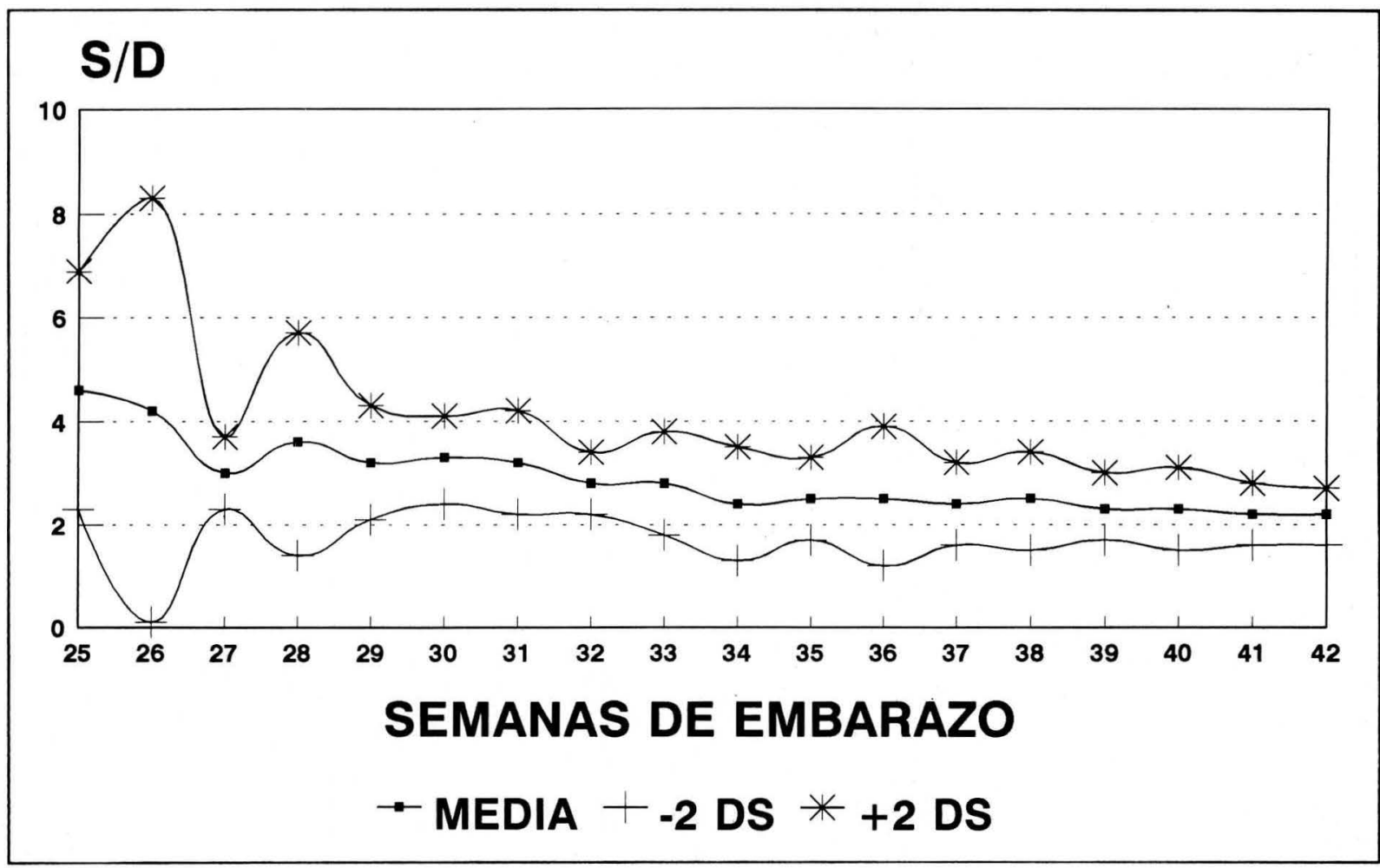

Evolución del índice de resistencia $S / D$ a lo largo del embarazo normal $(D S=$ Desviación Estándar $)$.

Figura 2

DOPPLER CONTINUO EN A. UMBILICAL - INDICE DE POURCELOT (GLOBAL)

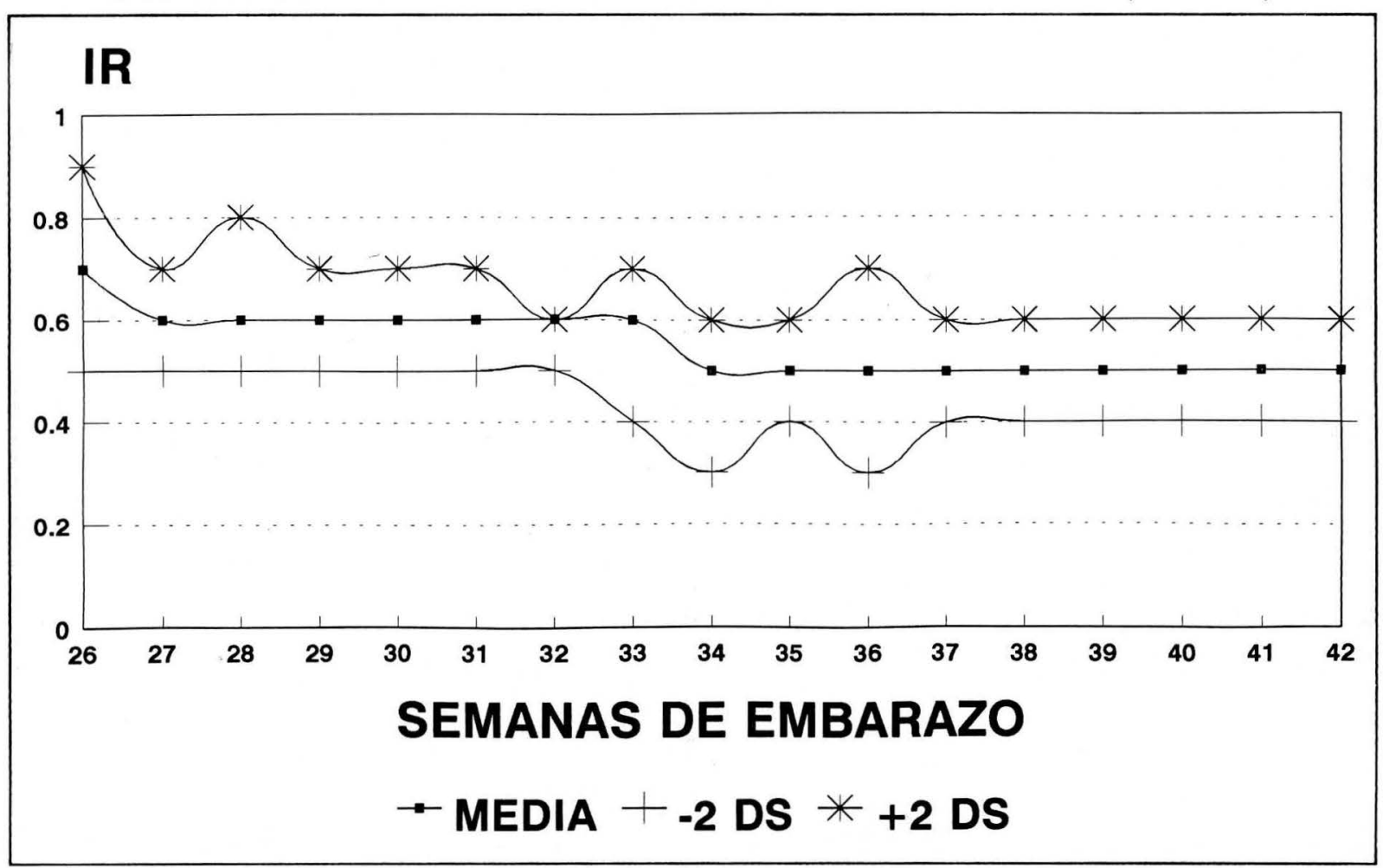

Evolución del índice de resistencia de Pourcelot a lo largo del embarazo normal ( $D S=$ Desviación Estándar). 
Figura 3

DOPPLER CONTINUO EN A. UMBILICAL - INDICE PULSATILIDAD (GLOBAL)

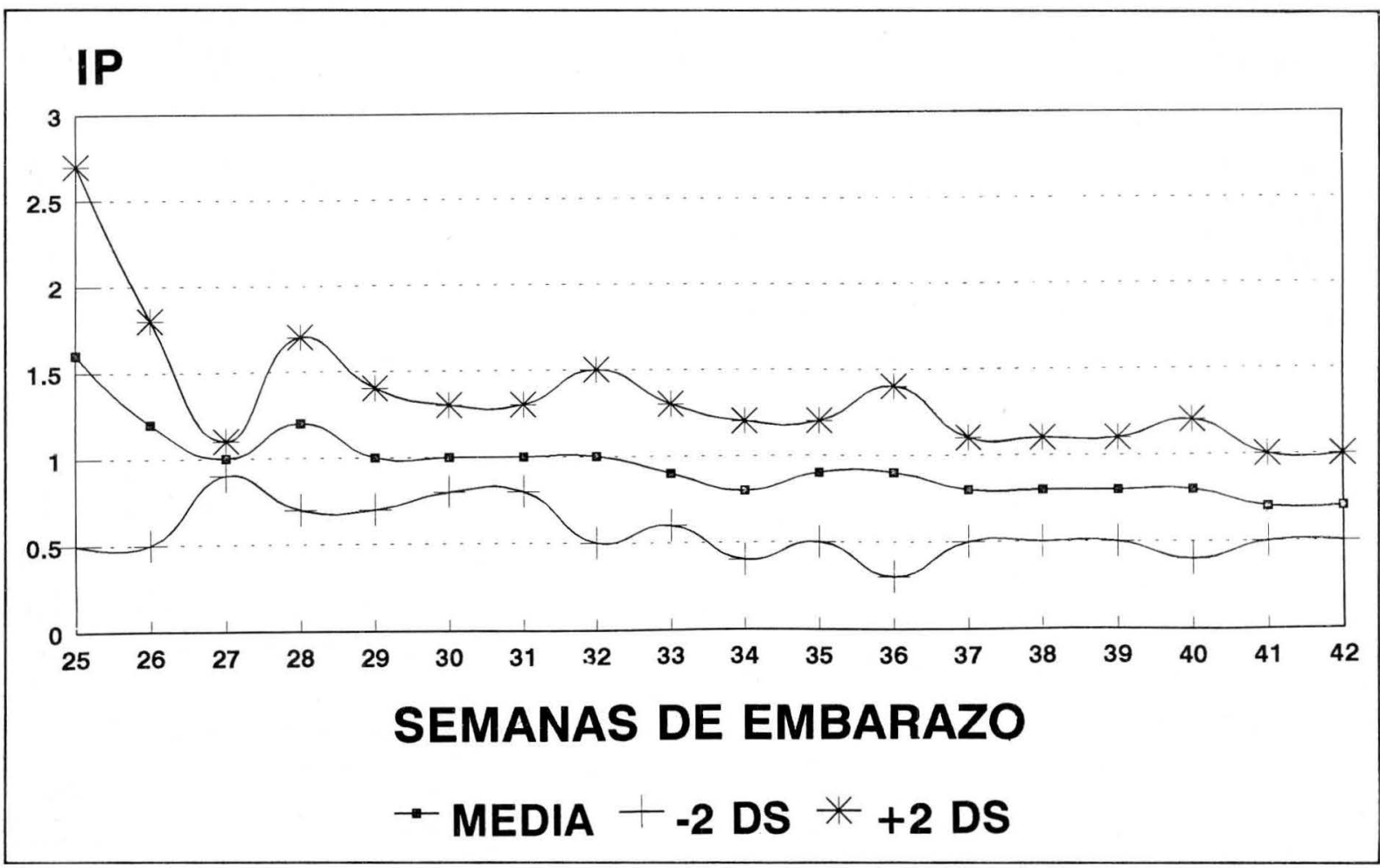

Evolución del índice de pulsatilidad a lo largo del embarazo normal (DS = Desviación Estándar $)$.

Figura 4

DOPPLER CONTINUO EN A. UMBILICAL - INDICE CONDUCTANCIA (GLOBAL)

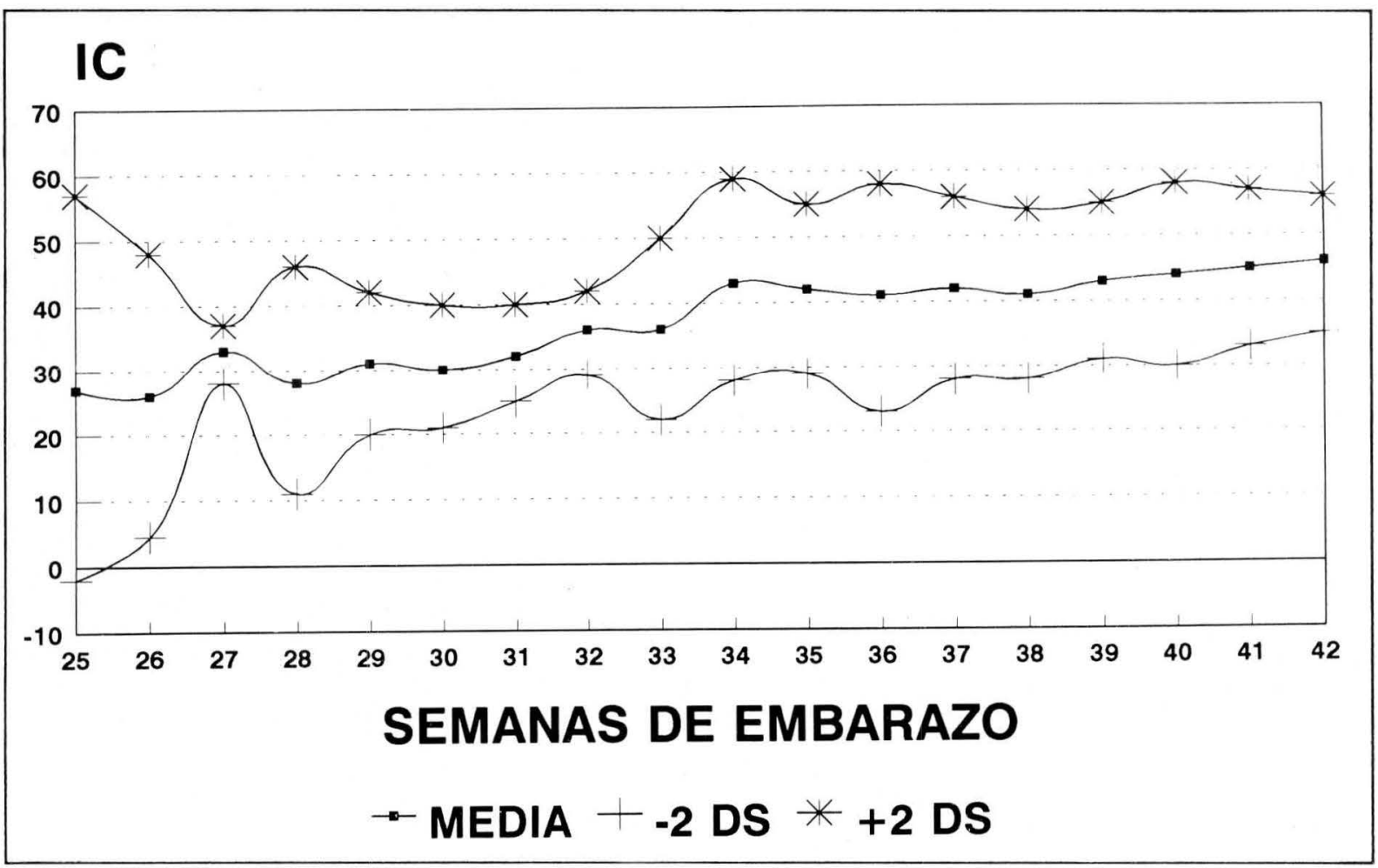

Evolución del índice de conductancia a lo largo del embarazo normal (DS = Desviación Estándar $)$. 
es la nuestra, estamos elaborando nuestras propias tablas de valores normales en los vasos fetales, que pensamos pueden ser de gran utilidad para nosotros y posiblemente para otros grupos nacionales que trabajen con el Doppler, ya que tal vez, al igual que sucedió con las tablas de crecimiento y peso fetal, los valores nacionales no sean similares a los extranjeros.

Creo, pues, que es conveniente que cada grupo de trabajo utilice tablas propias de valores normales de Doppler en cordón umbilical (29) y en todos los vasos fetales y maternos que se estudien por esta técnica, ya que metodológicamente es la forma más correcta de trabajar con las pacientes del medio correspondiente.

\section{Conclusiones}

1. Los Indices de Resistencia de Doppler Continuo en Arteria Umbilical disminuyen a medida que progresa el embarazo de curso normal, salvo el índice de conductancia que aumenta a lo largo del embarazo.

2. El sexo fetal no influye, ni condiciona, el descenso de los índices de resistencia con doppler continuo en arteria umbilical durantę el embarazo.

\section{Agradecimientos}

Mi agradecimiento a la cátedra de Bioestadística de la Facultad de Medicina de la Universidad de Málaga España, por la elaboración del trabajo estadístico.

\section{BIBLIOGRAFIA}

1. Carrera MJM et al. Doppler en Medicina Perinatal. Libro de Ponencias. XII Reunión Nacional de Medicina Perinatal. Valencia, España, Nov. 1990; 465-515.

2. Dawes GS. The Umbilical Circulation. In fetal and neonatal physiology. Year Book Medical Publishers 1968; 66-78.

3. Arabin B., Siebert M., Saling E. Prospective value of doppler measurement in uteroplacental and fetal vessels. A comparison of multiple parameters. Geburtsh Frauebh 1989; 49: 457-462.

4. Trudinger BJ., Cook C., Jones L et al. A comparison of fetal heart rate monitoring and umbilical artery waveforms in the recognition of fetal compromise. Br. J. Obstet. Gynecol. 1986; 93: 171-175.

5. Eik-Ness S., Marsal K., Kristoffersen K. Methodology and basic problems related to blood flow studies in the human fetus. Ultrasound. Med. Biol. 1984; 10: 329-337.

6. Trudinger BJ. The umbilical circulation. Seminars Perinatol 1987; 11: 311-321.

7. Gosling RG., Kling DH. Ultrasound Angiology. In Marcus AW, Adamson (eds): Arteries and Veins. Edinburgh, UK, ChurchillLivingtone, 1975; 61.

8. Fitzgerald DE., Drumm JE. Noninvasive measurement of fetal circulations using ultrasound: A New Method. Br. Med. J. 1977; 2: 1450.

9. Campbell S., Díaz Recasens J., Griffin Det al. New Doppler Technique for assessing uteroplacental blood flow. Lancet 1983; 1: 675.

10. Carrera JM., Mortera C., Torrents M. Efecto Doppler. Diagnóstico Prenatal. Salvat 1990; 24: 285-305.

11. Carrera JM., Alegre M., Torrents $\mathrm{M}$ et al. Evaluación de las resistencias vasculares uteroplacentarias mediante el análisis espectral de la onda de velocidad de flujo. Progr. Obstet. y Ginecol. 1986; 29(7): 397-404.

12. Carrera JM., Alegre M., Pérez-Ares C et al. Evaluación de las resistencias vasculares umbilicoplacentarias mediante el análisis espectral de la onda de velocidad de flujo. Progr. Obstet. y Ginecol. 1986; 29(6): 321-329.

13. Carrera JM., Alegre $M$ \& Torrents $M$. La fluxometría Doppler transplacentaria en los estados hipertensivos del embarazo. Gestosi 1987; I (ed). 89-94.

14. Gallo M., Chala JM., Gómez MO. Doppler versus NST en el diagnóstico del estado fetal intrauterino. II Congreso Nacional de Diagnóstico Prenatal, p. 218. Tenerife, España, 8-9 junio de 1989.

15. Gallo M., Gómez MO., Chala JM. Curvas de normalidad en el embarazo con Doppler continuo en cordón umbilical. XII Reunión Nacional Perinatal, p. 46. Valencia, España, 15-17 de noviembre de 1990.
16. Thompson RS., Trudinger BJ., Cook $\mathrm{CM}$ et al. Umbilical artery velocity waveforms: normal reference values for $\mathrm{A} / \mathrm{B}$ ratio and Pourcelot ratio. Br. J. Obstet. Gynecol. 1988; 55: 589-594.

17. Trudinger BJ., Warwick B., Giles MB et al. Flow velocity waveforms in the maternal uteroplacental and fetal umbilical placental circulations. Am. J. Obstet. Gynecol. 1985; 152: 155-163.

18. Stuart R., Dromm J., Fitzgerald Eet al. Fetal Blood velocity waveforms in normal pregnancy. Br. J. Obstet. Gynecol. 1980; 87: 780-785.

19. Fogaarty P., Beattie B., Harper A et al. Continuos wave doppler flow velocity waveforms from the umbilical artery in normal pregnancy. $\mathrm{J}$. Perinat. Med. Belfast. 1990; 18(1): 51-57.

20. Santonja Lucas JJ., Armero C., Navarro Piera E et al. Indices de velocimetría del flujo sanguíneo en la arteria umbilical en la segunda mitad de la gestación normal. Clin. Invest. Gin. Obs. 1990; 17(2): 42 47.

21. Schulman H., Fleischer A., Stern W et al. Umbilical velocity wave ratios in human pregnancy. Am. J. of Obstet. Gynecol. 1984; 148: 985-990.

22. Brar HS., Medearis AL., Devore GR., Platt LD. A Comparative study of fetal umbilical velocimetry whith continuous and pulsed-wave doppler ultrasonography in high-risk pregnancies. Relationship to outcome. Am. J. Obstet. Gynecol. 1989; 160: 375-378.

23. Mehalek KE., Berkowitz GS., Chitkara U et al. Comparison of continuos-wave and pulsed doppler S/D ratios of umbilical and uterine arteries. Obstet. Gynecol. 1988; 72: 603-606.

24. Stabile I., Sacks A., Campbell S. Doppler assessed uterine blood flow in the first trimester of pregnancy. Br. J. Obstet. Gynecol. 1986; 93: 1197.

25. Codesido J. Aplicaciones diagnósticas de la señal doppler acústica en Obstetricia y Ginecología. Tesis de Doctorado. Universidad de Santiago de Compostela, España, 1991.

26. Kofinas AD., Espeland M., Swain M et al. Correcting umbilical artery flow velocity waveforms for fetal heart rate is unnecessary. Am. J. Obstet. Gynecol. 1989; 160: 704-707.

27. Arbeille P., Huet L., Fignon A. Influence de la frequence cardiaque sur la determination des index circulatoires foeto-placentaires. IIIemes Journees Liegeoises de Gyn-Obstet. Liege, Belgiun, 1989.

28. Gudmundsson S \& Marsál K. Umbilical Artery and Uteroplacental blood flow velocity waveforms in normal pregnancy -A crossSectional Srudy. Acta Obstet. Gynecol. Scand. 1988; 67: 347-354.

29. Gómez MO. Curvas de Normalidad con Doppler Continuo en Arteria Umbilical durante el embarazo. Tesina de Licenciatura. Universidad de Málaga, España, 11 de noviembre de 1991 (Trabajo de Investigación para la S.C.O.G.). 